\title{
Surface states in photonic crystals
}

\author{
P. Vojtíšek ${ }^{1,2}$ and I. Richter ${ }^{2}$ \\ ${ }^{1}$ Institute of Plasma Physics AS CR, v.v.i., Centre TOPTEC, Sobotecká 1660, Turnov 511-01, Czech Republic \\ ${ }^{2}$ Czech Technical University in Prague, FNSPE, Department of Physical Electronics, Břehová 7, 115-19, Prague 1, \\ Czech Republic
}

\begin{abstract}
Among many unusual and interesting physical properties of photonic crystals $(\mathrm{PhC})$, in recent years, the propagation of surface electromagnetic waves along dielectric $\mathrm{PhC}$ boundaries have attracted considerable attention, also in connection to their possible applications. Such surfaces states, produced with the help of specialized defects on PhC boundaries, similarly to surfaces plasmons, are localized surfaces waves and, as such, can be used in various sensing applications. In this contribution, we present our recent studies on numerical modelling of surface states (SS) for all three cases of PhC dimensionality. Simulations of these states were carried out by the use of plane wave expansion (PWE) method via the MIT MPB package.
\end{abstract}

\section{Introduction}

Since their proposal in 1987 [1], photonic crystals $(\mathrm{PhC})$ or photonic band gap (PBG) structures [1-4], still represent very interesting and promising structures of artificial origin. Their main characteristics are given by spatially periodic variations of dielectric constant enabling in a sense such physical properties for light interactions as are exhibited by electrons in semiconductors. These properties are mainly represented with such effects as photonic bang gap (PBG) formation, prohibition of spontaneous emission and /or unusual laws of refraction / reflection,

Typically in theory, $\mathrm{PhC}$ are considered as ideal periodic crystals without presence of any defects, hence exhibiting corresponding Bloch modes. Contrary to this, realistically fabricated crystals usually exhibit some kind of defects which consequently alternate their properties. Figure 1 summarizes different types of defects which can be present in one-dimensional (1D), two-dimensional (2D) and three-dimensional (3D) $\mathrm{PhC}$. Yet another type of defects can exists in ideal crystals, this defect is given by a finite size of a crystal and can, under some conditions, lead to the excitation of a surfaces state (SS) propagating along the interface between a crystal and air (or more generally, background medium) [5-9], as a result of the interaction of $\mathrm{PhC}$ Bloch modes and outer plane wave modes. These surface states can exhibit very interesting properties leading to many potential applications in integrated photonics and optical sensing in particular [1], due to sharp resonant responses of these waves. Concerning the understanding of the physics of $\mathrm{SS}$, however, there is still lack of theoretical background and proper analysis of such intriguing phenomena.

Hence, the purpose of this paper, as a continuation of our previous studies, is to present and discuss our recent results on theoretical simulations of surface states as localized waves at the interface of $\mathrm{PhC}$ and air. Following that aim, this paper is organized as follows. After this introductory section, section 2 briefly presents the basics of surface states in $\mathrm{PhC}$. Next, in section 3, the paper concentrates further on our theoretical results, obtained via the plane wave expansion method (MIT MPB package) [10], of simulations of $\mathrm{SS}$ in $1 \mathrm{D}, 2 \mathrm{D}$, and 3D cases, respectively. Finally, section 4 summarizes the results and concludes the paper.

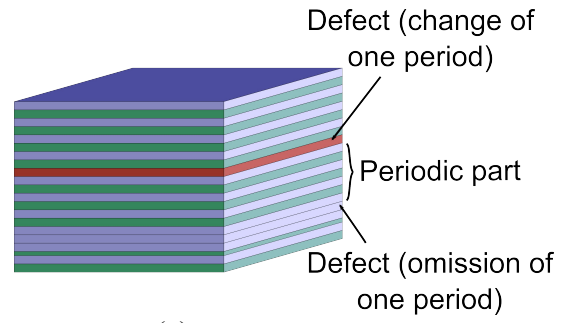

(a)

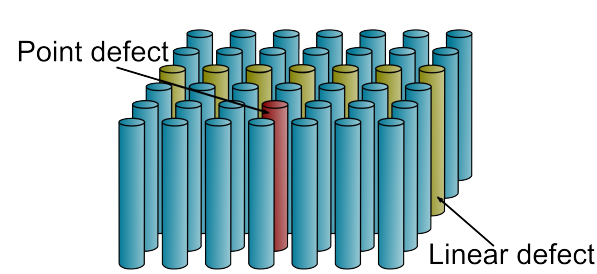

(b)

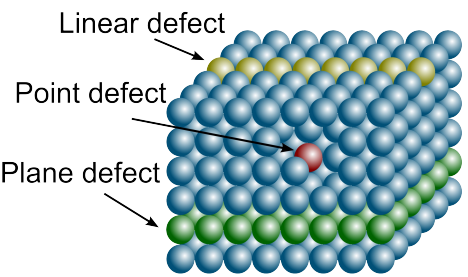

(c)

Fig. 1. Schematic view of a) $1 \mathrm{D} \mathrm{PhC}$ with corresponding structural defects, b) $2 \mathrm{D} \mathrm{PhC}$ with point and linear defects, and c) 3D $\mathrm{PhC}$ with tree types of defects (point, linear, plane).

This is an Open Access article distributed under the terms of the Creative Commons Attribution License 2.0, which permits unrestricted use, distribution, and reproduction in any medium, provided the original work is properly cited. Article available at http://www.epj-conferences.org or http://dx.dol.org/10.1051/epjcont/20134800030 


\section{Surface states at the boundary of PhC}

Since surface states represent localized waves at the interface of two media, they are in a sense similar to surface plasmons (SP) [3] which exist at the interface of a dielectric and metal. In contrast, in our case of SS, instead of a metallic component, a periodic modulation of the dielectric constant is considered, and connected to that, some kind of a PhC termination is required. Clearly, such termination breaks strict periodicity of the crystal. In fact, surface states can be exited at the surface of $\mathrm{PhC}$ if two conditions are met - (1) $\mathrm{PhC}$ has to be such that it pronounces a full photonic bang gap in the spectral region of interest (around this SS) and (2) light on the outer $\mathrm{PhC}$ side (air) has to be index-guided [1].

With respect to these two conditions one can divide surface states of light, which exist along the interface, into the two groups - (1) localized (decay, D) states, which exponentially decrease in an outer medium, and (2) extended (E) states, which propagate inside a medium. Further, one can specify four types of arrangements of these two states, which characterize possible states at both sides (and hence, along the boundary) of the interface simultaneously - EE, ED, DE, DD [2], where the first letter represents the state outside of a crystal and the second letter state inside a crystal. A proper surface state is the last one (i.e. DD), it is characterized by a decay of the fields on both sides of the interface. In other words, such DD surface states represent the Bloch states, and consequently, as such, do not couple to incoming / outgoing radiation.

Further, if one considers that a $\mathrm{PhC}$ already exhibits a full PBG in the spectral region of interest (this is mainly given by the periodicity and dielectric contrast), then we have to fulfil the condition for index-guiding of light outside of a crystal. Indeed, this condition can be met by appropriate modification of the last $\mathrm{PhC}$ period at the border with outside medium, i.e. via a change of inclination or a termination of this last period. Typically, the latter, i.e. the change in termination, is considered; it practically represents the elimination of some part of building blocks within the last period. Generally, a proper $\mathrm{PhC}$ termination is important for existence of a surface state, with no termination applied, there is no SS. Idea of a termination of $1 \mathrm{D} \mathrm{PhC}$ is presented schematically in figure 2a) together with a corresponding idea of exited surface state intensity at the interface is shown in figure 2b).

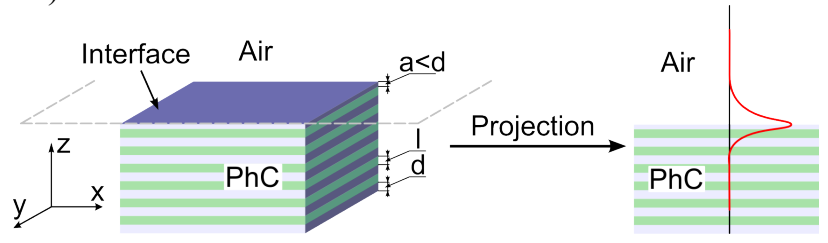

Fig. 2. a) Idea of termination of $1 \mathrm{D} \mathrm{PhC}$ and b) schematic distribution of a field intensity of a surface state along the boundary.

\section{Theoretical analysis of surface states}

In this section, we will present theoretical modelling and analysis of surface states at the interface of three different types (1D, 2D, and 3D) of a PhC and air. Simulations have been carried out by the use of PWE which was incorporated in the MPB package [9]. One, two and three dimensional simulations have been done to cover all three possible representations in which $\mathrm{PhC}$ is usually prepared.

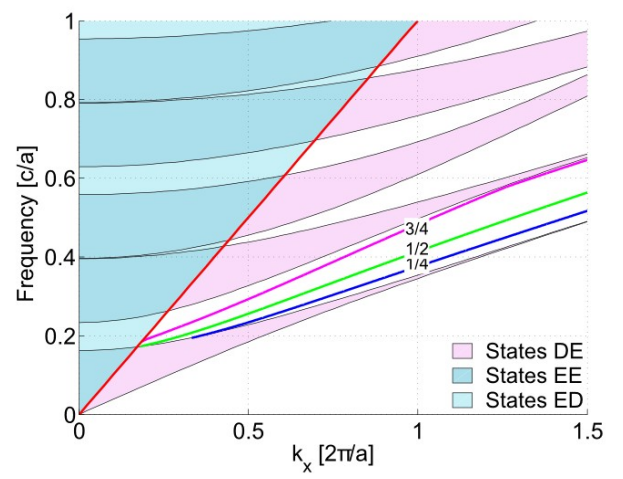

Fig. 3. Projected dispersion diagram for $1 \mathrm{D} \mathrm{PhC}$ with three SS for different values of the termination $(1 / 4,1 / 2,3 / 2)$ of the last layer of a PhC structure. $a$ denotes the period of a $\mathrm{PhC}$. Materials used were $\mathrm{Si}$ (layer dimension $0.36 a$ ) and $\mathrm{SiO}_{2}$ $(0.64 a)$.

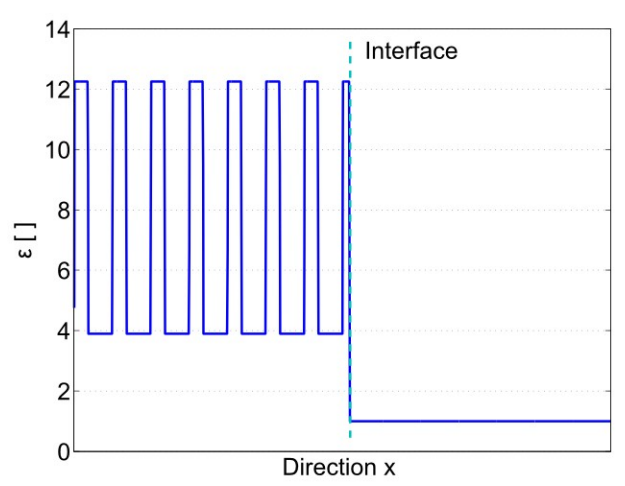

Fig. 4. Profile of 1D $\mathrm{PhC}\left(\mathrm{Si}: \mathrm{SiO}_{2}, 0.36 \mathrm{a}: 0.64 \mathrm{a}\right)$ with $1 / 2$ termination of the last layer indicated.

In the case of $1 \mathrm{D} \mathrm{PhC,}$ which is represented with a simple thin film multilayer, we have made the calculations for a structure consisting of periodic repetition of $\mathrm{Si}\left(\varepsilon=12.25\right.$ [11]) and $\mathrm{SiO}_{2}(\varepsilon=3.9)$. The width of a $\mathrm{Si}$ component (in fact, the thickness of a $\mathrm{Si}$ layer) was $0.36 a$ and the width of $\mathrm{SiO}_{2} 0.64 a$, where $a$ is the period of a structure. Figure 3 presents calculated projected dispersion diagram while figure 4 shows the corresponding profile of a dielectric constant with indicated interface, for the termination of $1 / 2$ of the last layer. In figure 3 , we can see three surface states for the three different types of termination $(1 / 4,1 / 2$ and $3 / 4)$ and four areas within the dispersion diagram which correspond to different behaviour of light. Above the light line (red line), there are blue and turquoise coloured areas present which correspond to EE and ED states, respectively. Below the light line, we can see DE states (pink colour) and PBG (white colour) areas. From the 
location of SS inside PBG, it is apparent that with a change of termination, the SS location is change as well.

In the case of 2D PhC, clearly, a spatial variability of possible structures is higher. For simulations, we have chosen two typical material $\mathrm{PhC}$ configurations - direct $\mathrm{PhC}$ and inverse $\mathrm{PhC}$, both with a triangular lattice (see figure 5).

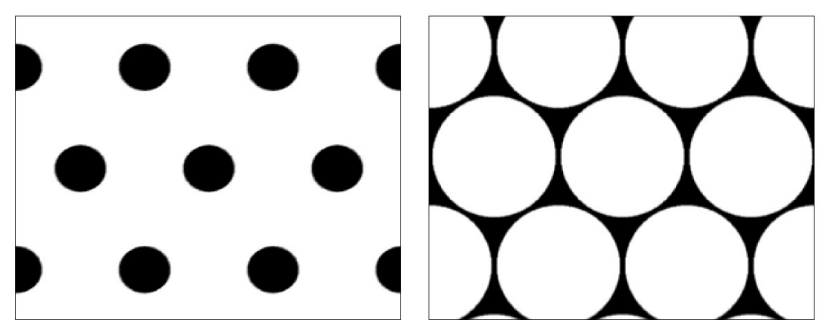

Fig. 5. Distribution of dielectric material for a) direct $(\mathrm{r}=0.2 a)$ and $\mathrm{b})$ inverse $(\mathrm{r}=0.48 a) 2 \mathrm{D} \mathrm{PhC}, a$ denotes the size of an elementary lattice vector.

In both cases, we have used as a base material Si (with $\varepsilon=12.25)$ and air $(\varepsilon=1)$ as a background material. For the direct $\mathrm{PhC}$, the structure consisted of dielectric rods with radius of $\mathrm{r}=0.2 a$, while for the inverse $\mathrm{PhC}$, the radius of air rods was chosen to be $\mathrm{r}=0.48 a$. The projected dispersion diagram (for TM polarization) is for a direct $\mathrm{PhC}$ shown in figure 6 . Then, figure 7 presents the dispersion diagram for both TM and TE polarizations for the case of an inverse $\mathrm{PhC}$. From each diagram (figures. 6 and 7), one can see that surface states can be indeed excited in such structures, for a properly chosen termination of the last period. In the case of an inverse $\mathrm{PhC}$, next, we have attempted to answer the following interesting question: Does a chosen termination of a crystal exist which would physically allow the existence of SS for both polarizations simultaneously? From the diagram shown in figure 8 , which represents the calculated dependence of the location of SS inside the PBG on the termination value chosen, it is apparent that, unfortunately, such termination does not exist, and, hence, SS for TM and TE polarization cannot be exited simultaneously for the same termination of a $\mathrm{PhC}$ boundary.

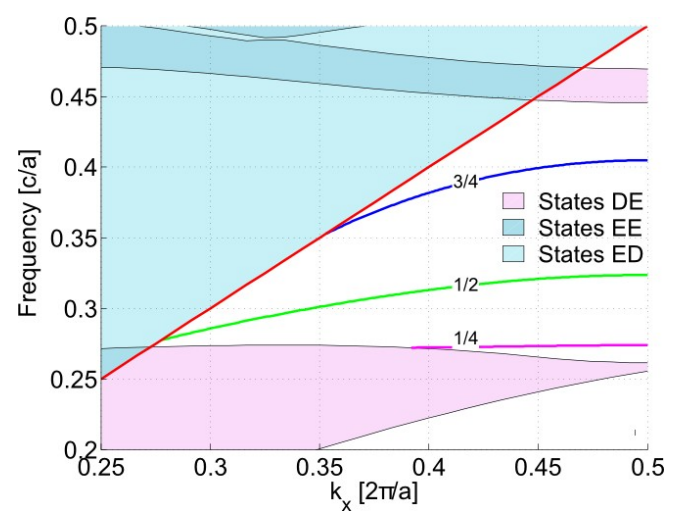

Fig. 6. Projected dispersion diagram of a direct $2 \mathrm{D} \mathrm{PhC}$ with existing SS indicated, for different termination values.
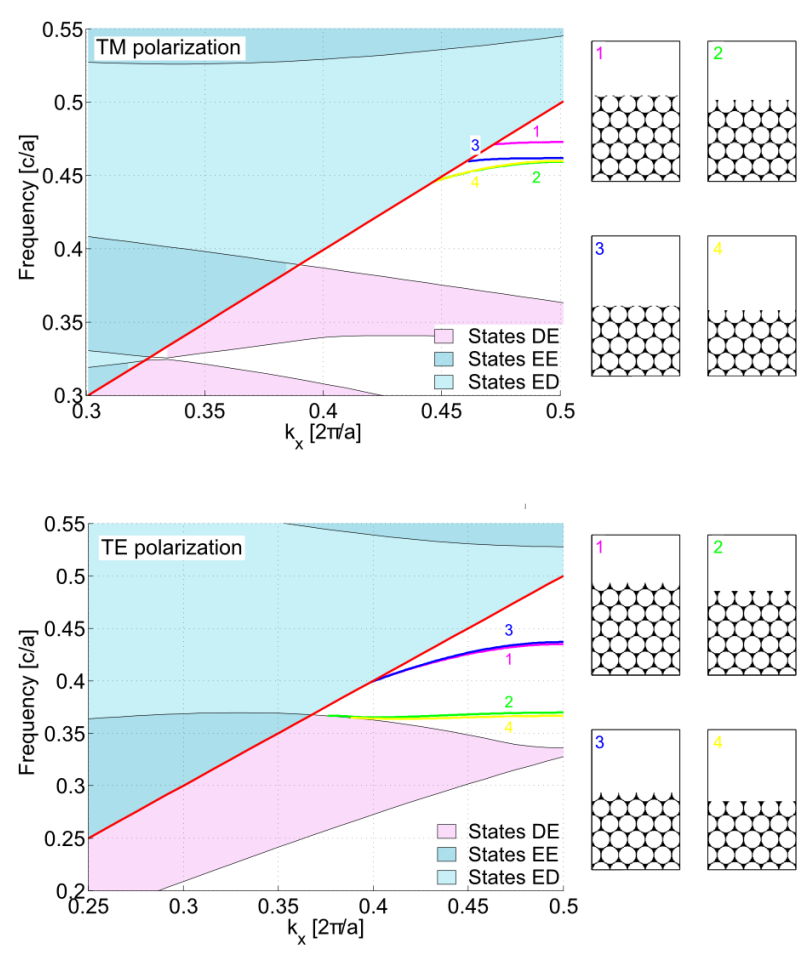

Fig. 7. Projected dispersion diagrams of an inverse 2D PhC: a) $\mathrm{TM}$ and b) TE polarization. Also, on the right-hand side, corresponding terminations user are shown.

Finally, for the three dimensional type of $\mathrm{PhC}$, we have chosen a simple opal FCC structure. On such an opaline based $\mathrm{PhC}$, we have studied possible existence of surface states. Here, the dielectric contrast was chosen to be 13 and the radius of an elementary ball $r=1 / \sqrt{ } 8 a$, where $a$ is the size of an elementary lattice. Figure 9 shows that, for these 3D structures, there is a possibility, for the chosen termination, to excite two surfaces states simultaneously. Additionally, in figure 10, one can see the corresponding distribution of the density of energy of the electric field. As can be seen, while one of the two possible SS is localized more inside the $\mathrm{PhC}$ opal, the other has more of its energy distributed outside of the crystal.

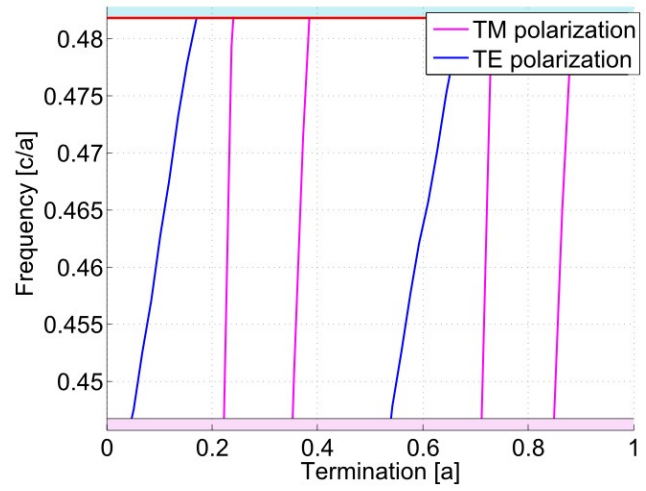

Fig. 8. Dependence of the spectral position of SS for $\mathrm{k}_{\mathrm{x}}=0.48(2 \pi / a)$ with respect to the termination for TM and TE polarizations, $a$ denotes the size of an elementary lattice vector. 


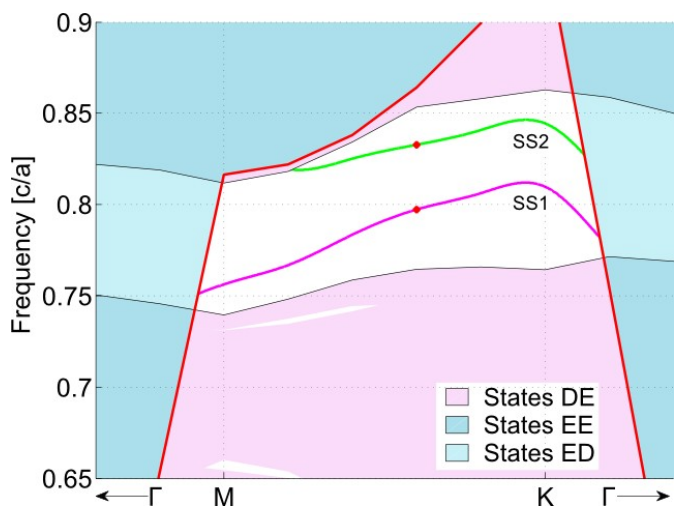

Fig. 9. Projected dispersion diagram for opaline $3 \mathrm{D} \mathrm{PhC}$ with two surface states present. Red dots denote position in $k$ space where density of energy of electric field was computed (see figure 10). (a)
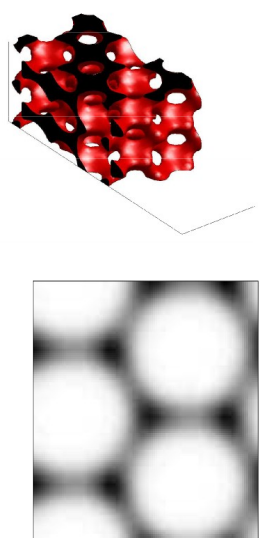

(d) (b)
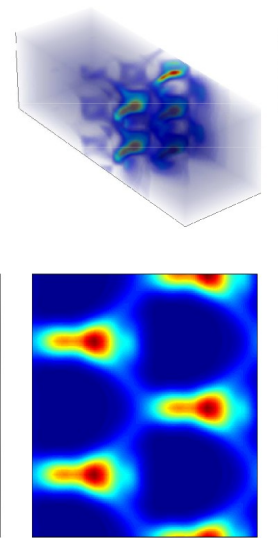

(e) (c)
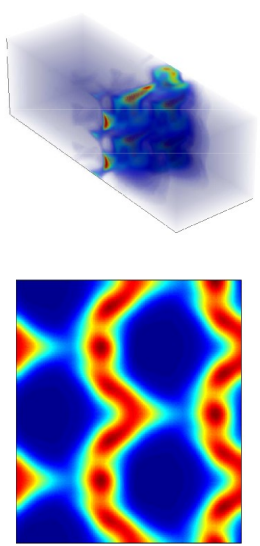

(f)
Fig. 10 . a) Distribution of a dielectric constant for 3D opal PhC considered, b) and c) distributions of the density of the electric energy of two possible surface states (SS1 and SS2 respectively, see figure 9), d), e) and f) cross-section of a), b) and c) along the interface.

\section{Conclusions}

In summary, we have presented our new results on the theoretical simulations of surface states as localized states in the presence of a special defect at a PhC boundary, i.e. the termination of the last $\mathrm{PhC}$ period. Our simulation results were based on the plane wave expansion method (represented with the MPB simulation package). Through simulations, we confirmed the existence of surfaces states on interfaces between a PhC crystal and air, for all three spatial dimensions. It was shown that different terminations of the last $\mathrm{PhC}$ period lead to different positions of SS inside a PBG, and hence, through different terminations, a proper tuning of spectral position of SS can be performed. In particular, for 3D opaline $\mathrm{PhC}$, two simultaneous surface states for the chosen termination were shown for the first time to exist. Although this existence was theoretically confirmed, practical realization of such samples can still be extremely difficult, clearly above the scope of simple self-assembled techniques efficiently used for the preparation of simple periodically arranged opaline $\mathrm{PhC}$. To conclude, such surface states, especially those existing for 2D PhC structures, can not only open up new possibilities for the design and operation of photonic structures in feeding / redistributing light applications, such as novel light couplers, emitters, etc., but also find their usage in sensing applications as interesting alternatives to surface plasmons.

\section{Acknowledgements}

This work was supported by the Czech Science Foundation (projects P205/10/0046 and P205/12/G118) and by the Ministry of Education COST project OC09038 and the European Regional Development Fund and the Ministry of Education, Youth and Sports of the Czech Republic in the Project No. CZ.1.05/2.1.00/03.0079: Research Center for Special Optics and Optoelectronic Systems (TOPTEC).

\section{References}

1. E. Yablonovitch, Phys. Rev. Letters 58, 2059 (1987)

2. J. D. Joannopoulos, S. G. Johnson, J. N. Winn, R. D. Meade, Photonic Crystals: Molding the Flow of Light, Princeton University Press, 2nd ed. (2008)

3. J.-M. Lourtioz, H. Benisty, V. Berger, J.-M. Gerard, D. Maystre, A. Tchelnokov, Photonic Crystals: Towards Nanoscale Photonic Devices, Springer, 1st ed. (2005)

4. K. Inoue, K. Ohtaka, Photonic crystals: physics, fabrication, and applications, Springer-Verlag, (2004)

5. A. P. Vinogradov, A. V. Dorofeenko, A. M. Merzlikin, A. A. Lisyansky, Physics - Uspekhi 53, 243 (2010)

6. A. P. Vinogradov, A. V. Dorofeenko, S. G. Erokhin, M. Inoue, A. A. Lisyansky, A. M. Merzlikin, A. B. Granovsky, Phys. Rev. B 74, 045128 (2006)

7. R. D. Meade, K. D. Brommer, A. M. Rappe, J. D. Joannopoulos, Phys. Rev. B 44, 10961 (1991)

8. K. Ishizaki and S. Noda, IEEE LEOS Annual Meeting LEOS '09, 24 (2009)

9. X. Zhang, L.-M. Li, Z.-Q. Zhang, C. T. Chan, Phys. Rev. B 63, 125114 (2001)

10. S. G. Johnson, J. D. Joannopoulos, Optics Express 8, 173 (2001)

11. C. Xi-Yao, L. Gui-Min, L. Jun-Jun, X. Xiao-Fu, J. Jun-Zhen, Q. Ze-Xuan, Q. Yi-Shen, L. Hui, Chinese Phys. Lett. 29, 014210 (2012) 